CARPATHIAN J. MATH.

Volume 37 (2021), No. 3,

Pages 477 - 487
Online version at https : //www. carpathian. cunbm. utcluj. ro/

Print Edition: ISSN 1584 - 2851; Online Edition: ISSN 1843 - 4401

DOI: https://doi.org/10.37193/CJM.2021.03.10

\title{
Strong convergence theorems for variational inequalities and fixed point problems in Banach spaces
}

\author{
M. O. NNAKWE ${ }^{1,2}$ and J. N. EZEORA ${ }^{3}$
}

\begin{abstract}
In this paper, using a sunny generalized non-expansive retraction which is different from the metric projection and generalized metric projection in Banach spaces, we present a retractive iterative algorithm of Krasnosel'skii-type, whose sequence approximates a common solution of a mono-variational inequality of a finite family of $\eta$-strongly-pseudo-monotone-type maps and fixed points of a countable family of generalized nonexpansive-type maps. Furthermore, some new results relevant to the study are also presented. Finally, the theorem proved complements, improves and extends some important related recent results in the literature.
\end{abstract}

\section{INTRODUCTION}

In this paper, we study the mono-variational inequality problem of Jouymandil and Moradloul [26] and $J$-fixed points for a countable family of generalized non-expansivetype maps in $L_{p}$ spaces, $2 \leq p<\infty$.

The first problem involving variational inequality was developed to solve equilibrium problems, precisely, the Signorini problem, posed in the year 1959, by Signorini [46], and was solved in the year 1963, by Fichera [22]. Hartman and Stampacchia studied the existence of solutions of variational inequality; see, for example, [24,35].

Variational inequality has been found to have numerous applications in many areas of mathematics, such as in partial differential equations, optimal control, optimization, mathematical programming and some other nonlinear problems; see, for example, $[3,5,6,8$, $15,16,23,53]$.

Numerous iterative methods have been proposed for solving variational inequality in the setting of real Hilbert spaces and more general Banach spaces; see, for example [9,32].

Let $\mathcal{Q}^{*}$ be the dual space of a real normed linear space, $\mathcal{Q}$. A map $A: \mathcal{Q} \rightarrow 2^{\mathcal{Q}}$ with domain $D(A)$ in $\mathcal{Q}$ is called accretive, if for all $u, v \in D(A)$, there exists $j(u-v) \in J(u-v)$ such that

$$
\left\langle\eta_{u}-\eta_{v}, j(u-v)\right\rangle \geq 0, \eta_{u} \in A u, \eta_{v} \in A v,
$$

where $J: \mathcal{Q} \rightarrow 2^{\mathcal{Q}^{*}}$ is the normalized duality map.

The map $A$ is called $m$-accretive, if $A$ is accretive and $R(I+s A)=\mathcal{Q}$, for all $s>0$, where $R(I+s A)$ denotes the range of $(I+s A)$ and $I$ is the identity map on $\mathcal{Q}$. In real Hilbert spaces, accretive maps are called monotone.

Accretive maps were introduced independently in the year 1967 by Browder [6] and Kato [31]. Interest in this class of maps stems mainly from their firm connection with the existence theory for nonlinear equations of evolution.

Received: 15.06.2020. In revised form: 20.03.2021. Accepted: 27.03.2021

2010 Mathematics Subject Classification. 47H09, 47H10, 47J25, 47J05, 47J20.

Key words and phrases. J-non-expansive maps, J-pseudo-monotone maps, J-variational inequalities, J-fixed points.

Corresponding author: M. O. Nnakwe; mon0002@auburn.edu; mondaynnakwe@gmail.com 
A fundamental problem in the study of accretive maps is the following:

$$
\text { find } u \in D(A) \text { such that } 0 \in A u \text {. }
$$

To study the inclusion (1.2) when $A$ is accretive, Browder [6] defined a self map $T$ on $\mathcal{Q}$ by $T:=(I-A)$ and called such a map pseudo-contractive. It is obvious that fixed points of $T$ correspond to solutions of the inclusion (1.2), assuming existence of a solution. For earlier and more recent results on the approximation of fixed points of pseudo-contractive maps, the reader may consult any of the following; [5, 13,33,38,41,44].

A map $A: \mathcal{Q} \rightarrow 2^{\mathcal{Q}^{*}}$ is called monotone, if for each $u, v \in \mathcal{Q}$, the following inequality holds:

$$
\left\langle\eta_{u}-\eta_{v}, u-v\right\rangle \geq 0, \eta_{u} \in A u, \eta_{v} \in A v .
$$

We recall in [43] that the sub-differential of a proper and convex function $f$, denoted by $\partial f$, is a monotone map, and for each $u \in \mathcal{Q}, 0 \in \partial f(u)$ if and only if $u$ is a minimizer of $f$. In particular, setting $\partial f \equiv A$, where $A$ is monotone, we have the fact that, $0 \in A u$. This also reduces to $A u=0$, where $A$ is single-valued.

Approximating zeros of such monotone maps is equivalent to finding a minimizer of $f$.

We observe that, the fixed point technique introduced by Browder [6], in the year 1967, for approximating zeros of accretive maps in a real Hilbert, for obvious reasons, is not applicable in this case, where $A$ is a monotone map from a real Banach space to its dual space.

Hence, there is the need to introduce and develop new techniques for approximating zeros of such monotone maps.

To approximate zeros of monotone maps, Chidume and Idu [12], in the year 2016, introduced a map, $T$, from a real normed space to its dual space, by $T:=(J-A)$, where $A: \mathcal{Q} \rightarrow 2^{\mathcal{Q}^{*}}$ is monotone. They called such a map $J$-pseudo-contractive.

Interest in $J$-pseudo-contractive maps stems mainly from their firm connection with the important class of nonlinear monotone maps (see also, [40]).

Let $T: \mathcal{Q} \rightarrow \mathcal{Q}^{*}$ be a map. An element $u \in \mathcal{Q}$ is called a $J$-fixed point of $T$, if

$$
T u=J u,
$$

where $J$ is single-valued in this case. Examples of $J$-fixed points include:

Example 1.1. [12] It is known (see e.g., Alber [3], pp.36) that in $l_{p}$ spaces, $1<p<\infty$,

$$
J u=\|u\|_{l_{p}}^{2-p} v \in l_{q}, v=\left\{\left|u_{1}\right|^{p-2} u_{1},\left|u_{2}\right|^{p-2} u_{2},\left|u_{3}\right|^{p-2} u_{3}, \cdots\right\}, u=\left\{u_{1}, u_{2}, \ldots\right\} \in l_{p} .
$$

For $1<q<p$, and any $\lambda \in \mathbb{R}$, set $\gamma_{p}:=\left(1+\frac{1}{2^{p}}\right)^{\frac{2-p}{p}}$ and define $T: l_{p} \rightarrow l_{q}$ by

$$
T\left(u_{1}, u_{2}, u_{3}, \ldots\right)=\left(\gamma_{p} u_{1}, \frac{\gamma_{p}}{2^{p-1}} u_{2}, 0,0,0, \ldots\right) .
$$

Let $u_{\lambda}=\left(\lambda, \frac{\lambda}{2}, 0,0, \ldots\right)$. Then, $T u_{\lambda}=\gamma_{p}\left(\lambda, \frac{\lambda}{2^{p}}, 0,0, \ldots\right)$ and $J u_{\lambda}=\gamma_{p}\left(\lambda, \frac{\lambda}{2^{p}}, 0,0, \ldots\right)$. Hence, $u_{\lambda} \in F_{J}(T)$.

Example 1.2. Let $A, T: L_{p}^{\mathbb{R}}([0,1]) \rightarrow L_{q}^{\mathbb{R}}([0,1]), 1<p<\infty, \frac{1}{p}+\frac{1}{q}=1$, be defined by

$$
A u(t):=(1+t) J u(t), \forall t \in[0,1] \text { and } T:=J-A .
$$

Clearly, $A$ is a monotone map. Hence, $u(t)=0$ is the fixed point of $T$, for all $t \in[0,1]$. 
We observe that, in a real Hilbert space and a strictly convex space, the notion of $J$-fixed point coincides with the classical definition of fixed point. However, if the space is not strictly convex, $J$ may fail to be one-to-one. Hence, the inverse of $J$ will not exist.

Therefore, approximating zeros of such monotone maps, is equivalent to approximating $J$-fixed points of $J$-pseudo-contractive maps, assuming existence of such zeros, which is also equivalent to finding minimizers of some convex functions.

In the year 2016, Chidume and Idu [12] studied this notion of fixed point and called it $J$ fixed point. This notion of fixed point has turned out to be very useful and applicable. For example, Chidume and Idu [12] studied the concept of $J$-fixed points and proved a strong convergence of a sequence, that approximates a $J$-fixed point of a $J$-pseudo-contractive map. As an application of their theorem, they proved a strong convergence of a sequence, that approximates a zero of an $m$-accretive map. They also applied their theorem on $J$ fixed points, to approximate solutions of Hammerstein integral equations. Nnakwe [36] in 2020 , also applied this concept of $J$-fixed point and proved a strong converge theorem for approximating a common solution of variational inequality and two convex minimization problems. For more recent works on $J$-fixed points; see, for example, [14,37,48,55].

Motivated by the ongoing research in this direction, it is our purpose in this paper to continue with the study of $J$-fixed points and some of it's applications. Here, we study a retractive iterative algorithm of Krasnosel'skii-type and prove a strong convergence of the sequence generated by the algorithm for approximating a common element in the set of solutions of mono-variational inequality of a finite family of $\eta$-strongly $J$-pseudo-monotone maps and fixed points of a countable family of generalized $J$-non-expansive maps in $L_{p}$ spaces, $2 \leq p<\infty$. Furthermore, we give some new definitions and lemmas relevant to the study. Finally, examples of $\eta$-strongly $J$-pseudo-monotone maps which are neither strongly $J$-monotone nor $J$-monotone and generalized $J$-non-expansive maps are constructed.

\section{PRELIMINARIES}

Let $\mathcal{Q}^{*}$ be the dual space of a smooth real Banach space $\mathcal{Q}$. Consider a map $\phi: \mathcal{Q} \times \mathcal{Q} \rightarrow \mathbb{R}$ defined by

$$
\phi(u, v)=\|u\|^{2}-2\langle u, J v\rangle+\|v\|^{2}, \text { for all } u, v \in \mathcal{Q} .
$$

This map which was introduced by Alber [3] will play a central role in the sequel. The following is a property of $\phi$ :

$$
\phi(u, w)=\phi(u, v)+\phi(v, w)+2\langle u-v, J v-J w\rangle, \text { for all, } u, v, w \in \mathcal{Q} .
$$

The following lemmas and definitions will be needed in the sequel.

Definition 2.1. Let $V: \mathcal{Q} \times \mathcal{Q}^{*} \rightarrow \mathbb{R}$ be defined by $V\left(u, u^{*}\right)=\|u\|^{2}-2\left\langle u, u^{*}\right\rangle+\left\|u^{*}\right\|$, for all $u \in \mathcal{Q}, u^{*} \in \mathcal{Q}^{*}$. It is easy to see that $V\left(u, u^{*}\right)=\phi\left(u, J^{-1} u^{*}\right), \forall u \in \mathcal{Q}, u^{*} \in \mathcal{Q}^{*}$.

Definition 2.2. [26] Let $M$ be a nonempty, closed and convex subset of $\mathcal{Q}$. The monovariational inequality is to find an element $u \in M$, such that $\langle\psi(u), J z-J u\rangle \geq 0, \forall z \in M$, where $\psi: M \rightarrow \mathcal{Q}$. The set of solutions of mono-variational inequality will be denoted by $V_{J}(\psi, M)$.

Definition 2.3. Let $\mathcal{Q}$ and $Y$ be real normed spaces, and $\psi: \mathcal{Q} \rightarrow Y$ be a map.

(a) $\psi$ is called $L$-Lipschitz, if there exists $L>0$ such that $\|\psi(u)-\psi(v)\| \leq L\|u-v\|, \forall u, v \in$ $\mathcal{Q}$.

It is known that in $L_{p}$ spaces, $p \geq 2, J_{p}$ is Lipschitz. 
Definition 2.4. [14] A map $T: M \rightarrow \mathcal{Q}^{*}$ is called generalized $J$-non-expansive, if $F_{J}(T) \neq \emptyset$ and $\phi\left(\left(J^{-1} o T\right) x, p\right) \leq \phi(x, p)$, for all $x \in M$, for all $p \in F_{J}(T)$.

NST-Condition. Let $\left\{S_{n}\right\}$ and $\Gamma$ be two families of generalized $J$-non-expansive maps from $M$ into $\mathcal{Q}^{*}$ such that $\cap_{n=1}^{\infty} F\left(S_{n}\right)=F(\Gamma) \neq \emptyset$, where $F\left(S_{n}\right)$ is the set of fixed points of $S_{n}$ and $F(\Gamma)$ is the set of fixed points of $\Gamma$. A sequence $\left\{S_{n}\right\}$ from $M$ to $\mathcal{Q}^{*}$ is said to satisfy the NST-condition with $\Gamma$, if for each bounded sequence $\left\{x_{n}\right\} \subset \mathcal{Q}, \lim \left\|J x_{n}-S_{n} x_{n}\right\|=0$ $\Longrightarrow \lim \left\|J x_{n}-S x_{n}\right\|=0, \forall S \in \Gamma$.

Lemma 2.1. [34] Let $M$ be a nonempty closed and convex subset of a smooth, strictly convex and reflexive Banach space $\mathcal{Q}$. Then, the following are equivalent.

(i) $M$ is a sunny generalized non-expansive retract of $\mathcal{Q}$

(ii) $M$ is a generalized non-expansive retract of $\mathcal{Q}$

(iii) $J M$ is closed and convex.

Lemma 2.2. [25] Let $M$ be a nonempty closed and convex subset of a smooth and strictly convex Banach space $\mathcal{Q}$ such that there exists a sunny generalized non-expansive retraction $R$ from $\mathcal{Q}$ onto $M$. Then, the following hold:

(i) $z=R x$ iff $\langle y-z, J z-J x\rangle \geq 0$, for all $y \in M$ and,

(ii) $\phi(x, R x)+\phi(R x, z) \leq \phi(x, z)$, for all $z \in M$.

Lemma 2.3. [29] Let $\mathcal{Q}$ be a uniformly convex and uniformly smooth real Banach space and $\left\{u_{n}\right\},\left\{v_{n}\right\}$ be sequences in $\mathcal{Q}$ such that either $\left\{u_{n}\right\}$ or $\left\{v_{n}\right\}$ is bounded. If $\lim \phi\left(u_{n}, v_{n}\right)=0$, then, $\lim \left\|u_{n}-v_{n}\right\|=0$.

Lemma 2.4. [11] Let $\mathcal{Q}=L_{p}, p \geq 2$. Then, this inequality holds:

$$
\|u-v\|^{2} \geq \phi(u, v)-p\|u\|^{2} \text {, for all } u, v \in \mathcal{Q} \text {. }
$$

Lemma 2.5. [10] Let $\mathcal{Q}=L_{p}, 1<p<2$. Then, this inequality holds:

$$
\|u+v\|^{2} \geq 2\|u\|^{2}+2\langle v, J u\rangle+c_{p}\|v\|^{2} \text {, for all } u, v \in \mathcal{Q} \text {, and some constant } c_{p}>0 \text {. }
$$

Lemma 2.6. [14] Let $\mathcal{Q}^{*}$ be the dual space of a uniformly convex and uniformly smooth real Banach space $\mathcal{Q}$. Let $M$ be a closed subset of $\mathcal{Q}$ such that $J M$ is closed and convex. Let $T$ be a generalized $J$-non-expansive map from $M$ to $\mathcal{Q}^{*}$ with $F_{J}(T) \neq \emptyset$. Then, $F_{J}(T)$ is closed and $J F_{J}(T)$ is closed and convex.

\section{MAIN RESULT}

Let $M$ be a nonempty, closed and convex subset of $\mathcal{Q}=L_{p}$ spaces, $1<p<\infty$, with dual space $\mathcal{Q}^{*}$. In this section, we present some new definitions, prove some new lemmas which are used to prove the main result of the section.

Definition 3.5. Let $\psi$ be a map from $M$ to $\mathcal{Q}$.

(a) $\psi$ is called $\eta$-strongly $J$-monotone, if there exists $\eta>0$ such that $\langle\psi(u)-\psi(v), J u-J v\rangle \geq$ $\eta\|u-v\|^{2}, \forall u, v \in M$,

(b) $\psi$ is called $J$-monotone, if $\langle\psi(u)-\psi(v), J u-J v\rangle \geq 0, \forall u, v \in M$,

(c) $\psi$ is called $\eta$-strongly $J$-pseudo-monotone, if there exists $\eta>0$, such that

$\langle\psi(u), J v-J u\rangle \geq 0 \Longrightarrow\langle\psi(v), J v-J u\rangle \geq \eta\|u-v\|^{2}, \forall u, v \in M$,

(d) $\psi$ is $J$-pseudo-monotone, if $\langle\psi(u), J v-J u\rangle \geq 0 \Longrightarrow\langle\psi(v), J v-J u\rangle \geq 0, \forall u, v \in M$.

In a real Hilbert space $H, J$ is the identity map on $H$ and $(a)-(d)$ of Definition 3.5 coincides with the usual definition of $(a)-(d)$ in the literature.

We observe that $(a) \Longrightarrow(b) \Longrightarrow(d)$ and $(a) \Longrightarrow(c) \Longrightarrow(d)$. The converse is not true. 
Lemma 3.7. Let $\mathcal{Q}$ be a uniformly convex and uniformly smooth real Banach space with dual space $\mathcal{Q}^{*}$. Let $M$ be a closed subset of $\mathcal{Q}$ such that $J M$ is closed and convex. Let $\psi$ be a J-pseudomonotone and Lipschitz map from $M$ to $\mathcal{Q}$ with $V_{J}(\psi, M) \neq \emptyset$. Then, $V_{J}(\psi, M)$ is closed and $J V_{J}(\psi, M)$ is closed and convex.

Proof. First, we prove that $V_{J}(\psi, M)$ and $J V_{J}(\psi, M)$ are closed. Obviously, $V_{J}(\psi, M)$ is closed. Let $\left\{v_{n}^{*}\right\} \subset J V_{J}(\psi, M)$ such that $v_{n}^{*} \rightarrow v^{*}$, for some $v^{*} \in \mathcal{Q}^{*}$. Since $J M$ is closed, we have that $v^{*} \in J M$. Hence, there exist $v \in M$ and $\left\{v_{n}\right\} \subset V_{J}(\psi, M)$ such that $v^{*}=J v$ and $v_{n}^{*}=J v_{n}, \forall n \in \mathbb{N}$. Utilizing the definition of $\psi$ and the fact that $J^{-1}$ is uniformly continuous on bounded subset of $\mathcal{Q}^{*}$, we have

$$
0 \leq \lim _{n \rightarrow \infty}\left\langle\psi J^{-1} v_{n}^{*}, J y-v_{n}^{*}\right\rangle=\left\langle\psi J^{-1} v^{*}, J y-v^{*}\right\rangle .
$$

Thus, we have that $v^{*}=J v \in J V_{J}(\psi, M)$. Hence, $J V_{J}(A, M)$ is closed.

Let $u^{*}, v^{*} \in J V_{J}(\psi, M)$. Then, $u^{*}=J u, v^{*}=J v \in J M$, for some $u, v \in M$. For $t, k \in(0,1)$, let $z^{*}=k u^{*}+(1-k) v^{*} \in J M$, and for any $y \in M$, we set $x_{t}^{*}=t J y+(1-t) z^{*}$. We compute as follows:

$$
\begin{aligned}
0=\left\langle\psi J^{-1} x_{t}^{*}, x_{t}^{*}-x_{t}^{*}\right\rangle & =\left\langle\psi J^{-1} x_{t}^{*}, J y-x_{t}^{*}\right\rangle-\left\langle\psi J^{-1} x_{t}^{*}, J y-x_{t}^{*}\right\rangle \\
& \leq(1-t)\left\langle\psi J^{-1} x_{t}^{*}, J y-z^{*}\right\rangle \leq\left\langle\psi J^{-1}\left(z^{*}+t\left(J y-z^{*}\right)\right), J y-z^{*}\right\rangle .
\end{aligned}
$$

Taking $\limsup _{t \downarrow 0}$ on (3.5); $\left\langle\psi J^{-1} z^{*}, J y-z^{*}\right\rangle \geq 0, \forall y \in M$. Thus, $z^{*} \in J V_{J}(\psi, M)$.

Remark 3.1. From Lemmas 2.6 and 3.7, we have that $J F_{J}(T)$ and $J V_{J}(\psi, M)$ are closed and convex. Since $J$ is one-to-one, we have that $J\left(F_{J}(T) \cap V_{J}(\psi, M)\right)=J F_{J}(T) \cap$ $J V_{J}(\psi, M)$. By Lemma 2.1, we have $F_{J}(T) \cap V_{J}(\psi, M)$ is a sunny generalized $J$-nonexpansive retract of $\mathcal{Q}$.

Lemma 3.8. Let $\mathcal{Q}=L_{p}$ spaces, $1<p<2$ or $p \geq 2$, and $\mathcal{Q}^{*}$ be the dual space of $\mathcal{Q}$. Then, for each $u, v \in \mathcal{Q}, u^{*} \in \mathcal{Q}^{*}$, the following inequalities hold:

$$
\begin{gathered}
V_{p}\left(u, u^{*}\right)+2\left\langle v, J u-u^{*}\right\rangle \leq V_{p}\left(u+v, u^{*}\right), \quad 1<p<2, \\
V_{p}\left(u, u^{*}\right)+2\left\langle v, J u-u^{*}\right\rangle \leq V_{p}\left(u+v, u^{*}\right)+(p-1)\|v\|^{2}, \quad p \geq 2 .
\end{gathered}
$$

Proof. For $1<p<2$; from Definition 2.1, property of $\phi_{,}\left(P_{1}\right)$ and Lemma 2.5, we have

$$
\begin{aligned}
V_{p}\left(u, u^{*}\right)+2\left\langle v, J u-u^{*}\right\rangle= & \phi(u, u+v)+\phi\left(u+v, J^{-1} u^{*}\right)-2\left\langle v, J(u+v)-u^{*}\right\rangle \\
& +2\left\langle v, J u-u^{*}\right\rangle \\
= & V_{p}\left(u+v, u^{*}\right)+\phi(u, u+v)+2\langle v, J u-J(u+v)\rangle \\
= & V_{p}\left(u+v, u^{*}\right)+\|u\|^{2}+2\langle v, J u\rangle-\|u+v\|^{2} \\
\leq & V_{p}\left(u+v, u^{*}\right)-c_{p}\|v\|^{2}, c_{p}>0 .
\end{aligned}
$$

Also, for $p \geq 2$, from inequality (3.8), Lemma 2.4 and interchanging $u, v \in \mathcal{Q}$, we have

$$
\begin{aligned}
V_{p}\left(u, u^{*}\right)+2\left\langle v, J u-u^{*}\right\rangle & =V_{p}\left(u+v, u^{*}\right)-\|u+v\|^{2}+\|u\|^{2}+2\langle v, J u\rangle \\
& \leq V_{p}\left(u+v, u^{*}\right)-\phi(v,-u)+p\|v\|^{2}+\|u\|^{2}+2\langle v, J u\rangle \\
& =V_{p}\left(u+v, u^{*}\right)+(p-1)\|v\|^{2} .
\end{aligned}
$$


We shall make the following assumptions.

$C_{1}$. The map $\psi_{i}$ is $\eta_{i}$-strongly- $J$-pseudo-monotone and $L$-Lipschitz on $M$, with $L>0$, $\eta_{i}>0, i=1,2, \cdots, N$.

$C_{2} \cdot\left\|\psi_{i}(v)\right\| \leq\left\|\psi_{i}(u)-\psi_{i}(v)\right\|$, for all $v \in M, u \in V_{J}\left(\psi_{i}, M\right), i=1,2, \cdots, N$.

\section{Convergence theorem in $L_{p}$ spaces, $p \geq 2$.}

Theorem 3.1. Let $M$ be a nonempty closed convex subset of $\mathcal{Q}=L_{p}, p \geq 2$, such that $J M$ is closed and convex. Let $\psi_{i}: M \rightarrow \mathcal{Q}, i=1,2, \cdots, N$ be a finite family of maps satisfying conditions $C_{1}$ and $C_{2}$. Let $S_{n}: M \rightarrow \mathcal{Q}^{*}, n=1,2, \ldots$ be a countable family of generalized $J$ non-expansive maps and $\Gamma$ be a family of closed and generalized $J$-non-expansive maps from $M$ to $\mathcal{Q}^{*}$ such that $F=:\left[\cap_{n=1}^{\infty} F_{J}\left(S_{n}\right)\right] \cap\left[\cap_{i=1}^{N} V_{J}\left(\psi_{i}, M\right)\right]=F_{J}(\Gamma) \cap\left[\cap_{i=1}^{N} V_{J}\left(\psi_{i}, M\right)\right] \neq \emptyset$. Let $\left\{v_{n}\right\}$ be a sequence generated iteratively by

$$
\left\{\begin{array}{l}
v_{1} \in M \\
y_{n}=R_{M}\left(v_{n}-\tau \psi_{n}\left(v_{n}\right)\right), \\
v_{n+1}=\beta v_{n}+(1-\beta) J^{-1} o S_{n}\left(y_{n}\right), \forall n \geq 1,
\end{array}\right.
$$

where $\psi_{n}=: \psi_{n}(\bmod N), \beta \in(0,1)$ and $\tau \in\left(0, \frac{2 \eta}{(2+p) L^{2}}\right)$, where $\eta:=\min \left\{\eta_{i}, i=1,2, \cdots, N\right\}$. Assume $\left\{S_{n}\right\}$ satisfies $N S T$-condition with $\Gamma$, then, $\left\{v_{n}\right\}$ converges strongly to $R_{F_{J}(\Gamma) \cap V_{J}(\psi, M)} v_{1}$; $R_{F_{J}(\Gamma) \cap V_{J}(\psi, M)}$ is the sunny generalized $J$-non-expansive retraction of $\mathcal{Q}$ onto $F_{J}(\Gamma) \cap V_{J}(\psi, M)$.

Proof. Let $u \in F$. Set $z_{n}=v_{n}-\tau \psi_{n}\left(v_{n}\right)$. By Lemma 3.8, we have

$$
\begin{aligned}
\phi\left(y_{n}, u\right) \leq & V\left(v_{n}-\tau \psi_{n}\left(v_{n}\right), J u\right) \\
\leq & V\left(v_{n}, J u\right)-2 \tau\left\langle\psi_{n}\left(v_{n}\right), J\left(v_{n}-\tau \psi\left(v_{n}\right)\right)-J u\right\rangle+\tau^{2}(p-1)\left\|\psi_{n}\left(v_{n}\right)\right\|^{2} \\
\leq & \phi\left(v_{n}, u\right)-2 \tau\left\langle\psi_{n}\left(v_{n}\right), J\left(v_{n}-\tau \psi_{n}\left(v_{n}\right)\right)-J v_{n}\right\rangle-2 \tau\left\langle\psi_{n}\left(v_{n}\right), J v_{n}-J u\right\rangle \\
& \quad+\tau^{2} p\left\|\psi_{n}\left(v_{n}\right)\right\|^{2} .
\end{aligned}
$$

since $u \in V_{J}\left(\psi_{n}, M\right)$, then, $\langle\psi(u), J x-J u\rangle \geq 0, \forall x \in M$. By $\eta$-pseudo- $J$-monotonicity of $\psi_{n}$ on $M$, we have that $\left\langle\psi_{n}(x), J x-J u\right\rangle \geq \eta\|x-u\|^{2}$. In particular, for $x=v_{n}$, we have that $\left\langle\psi_{n}\left(v_{n}\right), J v_{n}-J u\right\rangle \geq \eta\left\|v_{n}-u\right\|^{2}$. From inequality (3.10), conditions $C_{2}, C_{1}$ and the fact that $J$ is Lipschitz on $\mathcal{Q}$, we have

$$
\begin{aligned}
\phi\left(y_{n}, u\right) \leq & \phi\left(v_{n}, u\right)-2 \tau\left\langle\psi_{n}\left(v_{n}\right), J\left(v_{n}-\tau \psi_{n}\left(v_{n}\right)\right)-J v_{n}\right\rangle-2 \tau \eta\left\|v_{n}-u\right\|^{2} \\
& +\tau^{2} p\left\|\psi_{n}\left(v_{n}\right)-\psi_{n}(u)\right\|^{2} \\
\leq & \phi\left(v_{n}, u\right)+2 \tau^{2} L^{2}\left\|v_{n}-u\right\|^{2}-2 \tau \eta\left\|v_{n}-u\right\|^{2}+\tau^{2} L^{2} p\left\|v_{n}-u\right\|^{2} \\
= & \phi\left(v_{n}, u\right)-\tau\left(2 \eta-\tau L^{2}(2+p)\right)\left\|v_{n}-u\right\|^{2}
\end{aligned}
$$

$$
\begin{aligned}
\phi\left(v_{n+1}, u\right) & =\phi\left(\beta v_{n}+(1-\beta) J^{-1} o S_{n}\left(y_{n}\right), u\right) \\
& \leq \beta \phi\left(v_{n}, u\right)+(1-\beta) \phi\left(J^{-1} o S_{n} y_{n}, u\right)-\beta(1-\beta) g\left(\left\|v_{n}-J^{-1} o S_{n}\left(y_{n}\right)\right\|\right) \\
& \leq \beta \phi\left(v_{n}, u\right)+(1-\beta) \phi\left(y_{n}, u\right)-\beta(1-\beta) g\left(\left\|v_{n}-J^{-1} o S_{n}\left(y_{n}\right)\right\|\right) \\
& \leq \beta \phi\left(v_{n}, u\right)+(1-\beta)\left[\phi\left(v_{n}, u\right)-\tau\left(2 \eta-\tau L^{2}(2+p)\right)\left\|v_{n}-u\right\|^{2}\right] \\
& =\phi\left(v_{n}, u\right)-\tau(1-\beta)\left(2 \eta-\tau L^{2}(2+p)\right)\left\|v_{n}-u\right\|^{2} \leq \phi\left(v_{n}, u\right) .
\end{aligned}
$$

Hence, $\lim \phi\left(v_{n}, u\right)$ exists. Furthermore, $\left\{v_{n}\right\},\left\{y_{n}\right\}$ are bounded. 
Set $\Theta=\tau(1-\beta)\left(2 \eta-\tau L^{2}(2+p)\right)>0$, from inequality (3.13), we have

$$
\left\|v_{n}-u\right\| \leq \sqrt{\Theta^{-1}\left(\phi\left(v_{n}, u\right)-\phi\left(v_{n+1}, u\right)\right)} \rightarrow 0 \text { as } n \rightarrow \infty .
$$

Furthermore, using condition $C_{2}$ and Lipschitz property of $J$ on $\mathcal{Q}$, we have

$$
\begin{aligned}
\phi\left(y_{n}, v_{n}\right) & =\phi\left(v_{n}-\tau \psi_{n}\left(v_{n}\right), v_{n}\right)=V\left(v_{n}-\tau \psi_{n}\left(v_{n}\right), J v_{n}\right) \\
& \leq V\left(v_{n}, J v_{n}\right)-2 \tau\left\langle\psi_{n}\left(v_{n}\right), J\left(v_{n}-\tau \psi_{n}\left(v_{n}\right)-J v_{n}\right)\right\rangle+\tau^{2} p\left\|\psi_{n}\left(v_{n}\right)\right\|^{2} \\
& \leq \phi\left(v_{n}, v_{n}\right)+(2+p) \tau^{2} L^{2}\left\|v_{n}-u\right\|^{2} .
\end{aligned}
$$

From inequality (3.14), $\lim \phi\left(y_{n}, v_{n}\right)=0$. By Lemma 2.3, $\lim \left\|y_{n}-v_{n}\right\|=0$.

From inequalities (3.12) and (3.13), we have

$$
g\left(\left\|v_{n}-J^{-1} o S_{n}\left(y_{n}\right)\right\|\right) \leq(\beta(1-\beta))^{-1}\left[\phi\left(v_{n}, u\right)-\phi\left(v_{n+1}, u\right)\right] .
$$

Thus, $\lim g\left(\left\|v_{n}-J^{-1} o S_{n}\left(y_{n}\right)\right\|\right)=0$. By the property of $g, \lim \left\|v_{n}-J^{-1} o S_{n}\left(y_{n}\right)\right\|=0$.

Now,

$$
\left\|y_{n}-J^{-1} o S_{n}\left(y_{n}\right)\right\| \leq\left\|y_{n}-v_{n}\right\|+\left\|v_{n}-J^{-1} o S_{n}\left(y_{n}\right)\right\| \rightarrow 0(\text { as } n \rightarrow \infty) .
$$

By Lipschitz property of $J$ on $\mathcal{Q}$, we get that $\left\|J y_{n}-S_{n}\left(y_{n}\right)\right\| \rightarrow 0$ as $n \rightarrow \infty$. Since $\left\{S_{n}\right\}$ satisfies the NST-condition with $\Gamma$, then, $\lim \left\|J y_{n}-S y_{n}\right\|=0, \forall S \in \Gamma$. But $S$ is closed, hence, $u \in F_{J}(S)$.

\section{EXAMPLES}

We demonstrate the applicability of our result obtained in Theorem 3.1 to this example.

Example 4.3. Let $\mathcal{Q}=L_{p}([0,1]), p \geq 2$. Let $\alpha, \beta \in \mathbb{R}$ such that $0<\frac{\beta}{2}<\alpha \leq \beta \leq 1$.

Define $M_{\alpha}:=\left\{v \in L_{p}([0,1]):\|v\|_{L_{p}} \leq \alpha\right\} \subseteq \overline{B_{L_{p}}(0,1)}$ and $\psi_{\beta}: M_{\alpha} \rightarrow \mathcal{Q}$ be defined by

$$
\left(\psi_{\beta}(v)\right)(t):=(\beta-\|v\|) v(t) .
$$

Clearly, $V I\left(\psi_{\beta}, M_{\alpha}\right)=\{0\}$ and $u \in V I\left(\psi_{\beta}, M_{\alpha}\right)$ if and only if $u \in \psi_{\beta}^{-1}(0)$ satisfying conditions $C_{2}$. Also, let $u, v \in M_{\alpha}$, we have

$$
\begin{aligned}
\left\|\psi_{\beta}(u)-\psi_{\beta}(v)\right\| & =\left\|(\beta-\|u\|) v_{1}-(\beta-\|v\|) v\right\| \\
& \leq \beta\|u-v\|+\|u\|\|u-v\|+\|v\|\|u-v\| \leq(\beta+2 \alpha)\|u-v\| .
\end{aligned}
$$

Hence, $\psi_{\beta}$ is $L$-Lipschitz with $L=(\beta+2 \alpha)$.

Furthermore, let $u, v \in M_{\alpha}$ such that $\left\langle\psi_{\beta}(u), J v-J u\right\rangle \geq 0$. Since $\|u\|_{L_{p}} \leq \alpha \leq \beta$, this implies that $\langle u, J v-J u\rangle \geq 0$. Applying a result of $\mathrm{Xu}$ [54], we have

$$
\begin{aligned}
\left\langle\psi_{\beta}(v), J_{p} v-J_{p} u\right\rangle & =(\beta-\|v\|)\left\langle v, J_{p} v-J_{p} u\right\rangle \\
& \geq(\beta-\|v\|)\left(\left\langle v, J_{p} v-J_{p} u\right\rangle-\left\langle u, J_{p} v-J_{p} u\right\rangle\right) \\
& \geq(\beta-\alpha)\left\langle v-u, J_{p} v-J_{p} u\right\rangle \geq \frac{p^{-1} c_{p}}{2}(\beta-\alpha)\|v-u\|^{p}, c_{p}>0 .
\end{aligned}
$$

In particular, for $p=2, \psi_{\beta}$ is $\gamma$-strongly- $J$-pseudo-monotone with $\gamma=\frac{p^{-1} c_{p}}{2}(\beta-\alpha)>0$ satisfying condition $C_{1}$.

Hence, the sequence generated by Algorithm (3.9), converges strongly to $R_{F_{J}(\Gamma) \cap V_{J}(\psi, M)} v_{1}$. 
Let $S: M_{\alpha} \rightarrow \mathcal{Q}^{*}$ be defined by $S u=J u, \forall u \in M_{\alpha}$. Let $S_{n}: M_{\alpha} \rightarrow \mathcal{Q}^{*}$ be defined by

$$
S_{n} u=J\left(\alpha_{n} u+\left(1-\alpha_{n}\right) J^{-1} o S u\right), \forall n \geq 1, u \in M_{\alpha} \text { and } \alpha_{n} \in(0,1) .
$$

Clearly, $F_{J}\left(S_{n}\right)=F_{J}(S), \forall n \in \mathbb{N}$. Hence, $\underset{n \geq 1}{\cap} F_{J}\left(S_{n}\right)=F_{J}(S)$. For any $u \in M, v \in$ $F_{J}\left(S_{n}\right)$, we have

$$
\begin{aligned}
\phi\left(J^{-1} o S_{n} u, v\right) & =\phi\left(\alpha_{n} u+\left(1-\alpha_{n}\right) J^{-1} o S u, v\right) \\
& \leq \alpha_{n} \phi(u, v)+\left(1-\alpha_{n}\right) \phi\left(J^{-1} o S u, v\right) \\
& =\alpha_{n} \phi(u, v)+\left(1-\alpha_{n}\right) \phi(u, v)=\phi(u, v) .
\end{aligned}
$$

Hence, $\left\{S_{n}\right\}$ is a generalized $J$-non-expansive map, where the map $\phi$ is the Alber's functional.

Let $\left\{u_{n}\right\}$ be a bounded sequence in $M_{\alpha}$ such that $\lim \left\|J u_{n}-S_{n} u_{n}\right\|=0$. Since $\left\{u_{n}\right\}$ is bounded, then, $\left\{J^{-1} o S u_{n}\right\}$ is bounded. Using the definition of $S_{n}$, we have

$$
\left\|u_{n}-J^{-1} o S u_{n}\right\|=\frac{1}{1-\beta_{n}}\left\|u_{n}-J^{-1} o S_{n} u_{n}\right\| \leq 2\left\|u_{n}-J^{-1} o S_{n} u_{n}\right\| .
$$

Since $\lim \left\|J u_{n}-S_{n} u_{n}\right\|=0$ and the fact that $J^{-1}$ and $J$ are uniformly continuous on bounded subsets of $\mathcal{Q}^{*}$ and $\mathcal{Q}$, respectively, we have that $\lim \left\|J u_{n}-S u_{n}\right\|=0$, which implies that $\left\{S_{n}\right\}$ satisfies $N S T$-condition with $S$.

Hence, the sequence generated by Algorithm (3.9), converges strongly to $R_{F_{J}(\Gamma) \cap V_{J}(\psi, M)} v_{1}$.

Example 4.4. Let $M=[0,1]$ and $\psi: M \subset \mathbb{R} \rightarrow \mathbb{R}$ be given by $\psi(v)=(2-v), \forall v \in M$. Clearly, $V I(\psi, M)=\{0\}$ and $\psi$ is 1-Lipschitz. Furthermore, suppose that $u, v \in M$ such that $\langle\psi(u), v-u\rangle \geq 0$. Since $u \in[0,1]$, this implies that $u<v$. Thus,

$$
\langle\psi(v), v-u\rangle=\langle 2-v, v-u\rangle \geq|v-u| \geq|v-u|^{2}, \forall v \in M,
$$

which implies that $\psi$ is 1-strongly- $J$-pseudo-monotone. Moreover, $\psi$ is neither strongly$J$ - monotone nor $J$-monotone. To see this, choose $u=\frac{1}{2}$ and $v=1$.

\section{DISCUSSION}

Theorem 3.1 which approximate a common solution of a finite family of mono-variational inequality problems and a common fixed points of a countable family of generalized-J-nonexpansive maps complement and extend important recent results in the literature, in particular, the result of Khanh, [32] from a Hilbert space to $L_{p}$ spaces, $2 \leq p<\infty$, respectively. Furthermore, the theorems proved are analogue of the result of Khanh, [32] in that if $\mathcal{D}=H$, a real Hilbert space, the normalized duality map is the identity on $\mathcal{D}$. Hence, the both theorems coincide. Finally, the class of $\eta$-strongly J-pseudo-monotone maps considered, contains the class $J$-monotone maps studied in [26].

Acknowledgment. The authors appreciate the support of their institutions. They are also grateful to the anonymous referees and the editor for their valuable comments and suggestions that helped to improve the quality of this paper.

Note added by Editors. To illustrate how interesting is the topic of the paper, we also included at the end of authors' list of References a selective list of some recent related published papers, see [7]- [49]. 


\section{REFERENCES}

[1] Abass, H. A.; Izuchukwu, C.; Mewomo, O. T. Viscosity approximation method for modified split generalized equilibrium and fixed point problems. Rev. Un. Mat. Argentina 61 (2020), no. 2, 389-411.

[2] Abass, H. A.; Mebawondu, A. A.; Mewomo, O. T. Convergence analysis of quasi-variational inclusion and fixed point problems of finite family of certain nonlinear mappings in Hilbert spaces. Thai J. Math. 18 (2020), no. 3, 1565-1579.

[3] Alber, Ya. Metric and generalized projection operators in Banach spaces: Properties and applications. A. G. Kartsatos (Ed.), Theory and Applications of Nonlinear Operators of Accretive and Monotone Type, in: Lecture Notes Pure Appl. Math., 178 (1996), 15-50.

[4] Alansari, M.; Ali, R.; Farid, M. Strong convergence of an inertial iterative algorithm for variational inequality problem, generalized equilibrium problem, and fixed point problem in a Banach space. J. Inequal. Appl. 2020, Paper No. 42, 22 pp.

[5] Berinde, V. Iterative Approximation of Fixed points, Lecture Notes in Mathematics. Springer, London, UK. (2007).

[6] Browder, F. E. Nonlinear mappings of nonexpansive and accretive-type in Banach spaces. Bull. Amer. Math. Soc. 73 (1967), 875-882.

[7] Cai, G.; Dong, Q.-L.; Peng, Y. Strong convergence theorems for inertial Tseng's extragradient method for solving variational inequality problems and fixed point problems. Optim. Lett. 15 (2021), no. 4, 1457-1474.

[8] Censor, Y.; Gibali, A. ; Reich, S. The subgradient extragradient method for solving variational inequalities in Hilbert space. J. Optim. Theory Appl. 148 (2011), 318-335.

[9] Censor, Y.; Gibali, A.; Reich, S. Two extensions of Korpelevich's extragradient method for solving the variational inequality problem in Euclidean space. Optimization 61 (2010), 1119-1132.

[10] Chidume, C. E. Geometric Properties of Banach Spaces and Nonlinear Iterations, vol. 1965. Springer-Verlag London, Ltd., London, 2009.

[11] Chidume, C. E.; Bello, A. U. An iterative algorithm for approximating solutions of Hammerstein equations with monotone maps in Banach spaces. Appl. Math Compt. 313 (2017), 408-417.

[12] Chidume, C. E.; Idu, C. E. Approximation of zeros of bounded maximal monotone maps, solutions of Hammerstein integral equations and convex minimization problems. Fixed Point Theory Appl. (2016) No. 97, doi.org:10.1186/s13663-016-0582-8.

[13] Chidume, C. E.; Mutangadura, S. A. An example on the Mann iteration method for Lipschitz pseudocontrations. Proc. Amer. Math. Soc. 129 (2001), No. 8, 2359-2363.

[14] Chidume, C. E.; Nnakwe, M. O. A new Halpern-type algorithm for a generalized mixed equilibrium problem and a countable family of generalized-J-nonexpansive maps, with applications. Carpathian J. Math. 34 (2018), no. 2, 191-198.

[15] Chidume, C. E.; Nnakwe, M. O., Iterative algorithms for split variational inequalities and generalized split feasibility problems, with applications. J. Nonlinear Var. Anal. 2 (2019), 127-140.

[16] Chidume, C. E.; Nnyaba, U. V.; Romanus, O. M.; Ezea, C. G. Convergence theorems for strictly Jpseudocontractions with application to zeros of Gamma-inverse strongly monotone maps. PanAmer. Math. J. 26 (2016), 57-76.

[17] Guan, J.-L.; Ceng, L.-C.; Hu, B. Strong convergence theorem for split monotone variational inclusion with constraints of variational inequalities and fixed point problems. J. Inequal. Appl. 2018, Paper No. 311, 29 pp.

[18] Darvish, V. A strong convergence theorem for finding a common fixed point of a finite family of Bregman nonexpansive mappings in Banach spaces which solves a generalized mixed equilibrium problem. Boll. Unione Mat. Ital. 9 (2016), no. 4, 421-434.

[19] Deepho, J.; Thounthong, P.; Kumam, P.; Phiangsungnoen, S. A new general iterative scheme for split variational inclusion and fixed point problems of k-strict pseudo-contraction mappings with convergence analysis. J. Comput. Appl. Math. 318 (2017), 293-306.

[20] Djafari-Rouhani, B.; Kazmi, K. R.; Farid, M. Common solutions to some systems of variational inequalities and fixed point problems. Fixed Point Theory 18 (2017), no. 1, 167-190.

[21] Farid, M.; Kazmi, K. R. A new mapping for finding a common solution of split generalized equilibrium problem, variational inequality problem and fixed point problem. Korean J. Math. 27 (2019), no. 2, $297-327$.

[22] Fichera, G. Problemi elastostatici con vincoli unilaterali (unilateral constraints); il problema di Signorini con ambigue condizioni al contorno (ambiguous boundary conditions). Mem. Accad. Naz. Lincei. 7 (1964), 91-140.

[23] Gibali, A.; Reich, S.; Zalas, R. Iterative methods for solving variational inequalities in Euclidean space. J. Fixed Point Theory Appl. 17 (2015), 775-811.

[24] Hartman, P.; Stampacchia, G. On some non-linear elliptic differential-functional equations. Acta Math. 115 (1966), 271-310.

[25] Ibaraki, T.; Takahashi, W. A new projection and convergence theorems for the projections in Banach spaces. J. Approx. Theory 149 (2007), 1-14. 
[26] Jouymandil, Z.; Moradloul, F. Retraction algorithms for solving variational inequalities, pseudo-monotone equilibrium problems, and fixed-point problems in Banach spaces. Numer. Algor. 78 (2018), 1153-1182.

[27] Jolaoso, L. O.; Taiwo, A.; Alakoya, T. O.; Mewomo, O. T. A self adaptive inertial subgradient extragradient algorithm for variational inequality and common fixed point of multivalued mappings in Hilbert spaces. Demonstr. Math. 52 (2019), no. 1, 183-203.

[28] Jolaoso, L. O.; Aphane, M. Strong convergence inertial projection and contraction method with self adaptive stepsize for pseudomonotone variational inequalities and fixed point problems. J. Inequal. Appl. 2020, Paper No. 261, 22 pp.

[29] Kamimura, S.; Takahashi, W. Strong convergence of a proximal-type algorithm in a Banach space. SIAM J. Optim. 13 (2002), no. 3, 938-945.

[30] Kangtunyakarn, A. The split combination of variational inequalities for fixed point theory of a finite family of nonspreading mappings. Rev. R. Acad. Cienc. Exactas Fís. Nat. Ser. A Mat. RACSAM 111 (2017), no. 4, 1163-1175.

[31] Kato, T. Nonlinear semi-groups and evolution equations. J. Math. Soc. Japan 19 (1967), no. 4, 508-520.

[32] Khanh, P. D. A new extragradient method for strongly pseudomonotone variational inequalities. Numer. Funct. Anal. Optim. 37 (2016), 1131-1143.

[33] Kirk, W. A. On local expansions and accretive mappings. Internat. J. Math. Math. Sci. 6 (1983), 419-429.

[34] Kohsaka, F.; Takahashi, W. Generalized nonexpansive retractions and a proximal-type algorithm in Banach spaces. J Nonlinear Convex Anal. 8 (2007), no. 2, 197-209.

[35] Lions, J. L.; Stampacchia, G. Variational inequalities. Comm. Pure Appl. Math. 20 (1967), 493-519.

[36] Nnakwe, M. O. An algorithm for approximating a common solution of Variational inequality and convex minimization problems. Optimization (2020), doi.org/10.1080/02331934.2020.1777995.

[37] Nnakwe, M. O.; Okeke, C. C. A common solution of generalized mixed equilibrium problems and a common fixed point of pseudocontractive-type maps. J. Appl. Math. Comput. (2020), doi.org/10.1007/s12190020-01457-x.

[38] Ofoedu, E. U.; Odumegwu J.; Zegeye, H.; Shahzad, N. An algorithm for finding common solutions of various problems in nonlinear operator theory. Fixed Point Theory Appl. (2014), no. 9, doi.org/10.1186/16871812-2014-9.

[39] Ogbuisi, F. U. A self-adaptive subgradient extragradient algorithm for variational inequality problems and fixed point problems in Banach spaces. Aust. J. Math. Anal. Appl. 17 (2020), no. 2, Art. 15, 17 pp.

[40] Reich, S. Constructive techniques for accretive and monotone operators. Applied Nonlinear Analysis (Proc. Third Internat. Conf., Univ. Texas, Arlington, Tex., 1978), pp. 335-345, Academic Press, New York-London, 1979.

[41] Reich, S. Extension problems for accretive sets in Banach spaces. J. Functional Analysis 26 (1977), 378-395.

[42] Rizvi, S. H. A strong convergence theorem for split mixed equilibrium and fixed point problems for nonexpansive mappings. J. Fixed Point Theory Appl. 20 (2018), no. 1, Paper No. 8, 22 pp.

[43] Rockarfellar, R. T., Convex Analysis. Princeton University Press, 242 (1970), ISBN 0-691-08069-0.

[44] Schu, J. Iterative construction of fixed points of asymptotically nonexpansive mappings. J. Math. Anal. Appl. 158 (1991), no. 2, 407-413.

[45] Shehu, Y.; Agbebaku, D. F. On split inclusion problem and fixed point problem for multi-valued mappings. Comput. Appl. Math. 37 (2018), no. 2, 1807-1824.

[46] Signorini, A. Questioni di elasticità nonlinearizzata semilinearizzata. Rend. Mat. 18 (1959), 1-45.

[47] Siriyan, K.; Kangtunyakarn, A. Algorithm method for solving the split general system of variational inequalities problem and fixed point problem of nonexpansive mapping with application. Math. Methods Appl. Sci. 41 (2018), no. 17, 7766-7788.

[48] Su, Y.; Xu, H. K. A duality fixed point theorem and applications. Fixed Point Theory 13 (2012), no. 1, $259-265$.

[49] Sunthrayuth, P.; Kumam, P. The resolvent operator techniques with perturbations for finding zeros of maximal monotone operator and fixed point problems in Hilbert spaces. Thai J. Math. 14 (2016), Special issue, $1-21$.

[50] Takahashi, W. A general iterative method for split common fixed point problems in Hilbert spaces and applications. Pure Appl. Funct. Anal. 3 (2018), no. 2, 349-369.

[51] Takahashi, W.; Wen, C.-F.; Yao, J.-C. Split common fixed point problems and hierarchical variational inequality problems in Hilbert spaces. J. Nonlinear Convex Anal. 18 (2017), no. 5, 777-797.

[52] Thong, D. V. Viscosity approximation methods for solving fixed-point problems and split common fixedpoint problems. J. Fixed Point Theory Appl. 19 (2017), no. 2, 1481-1499.

[53] Yao, Y.; Marino, G.; Muglia, L. A modified Korpelevich's method convergent to the minimum-norm solution of a variational inequality. Optimization 63 (2014), 559-569.

[54] Xu, H. K. Inequalities in Banach spaces with applications. Nonlinear Analysis: Theory, Methods and Applications 16 (1991), no. 12, 1127-1138. 
[55] Zegeye, H. Strong convergence theorems for maximal monotone mappings in Banach spaces. J. Math. Anal. Appl. 343 (2008), 663-671.

${ }^{1}$ Department of Mathematics and Statistics, Auburn University, Alabama, AL 36849, USA

${ }^{2}$ Mathematics Institute, African University of Science and Technology, Abuja, Nigeria

E-mail address: mon0002@auburn.edu

E-mail address: mondaynnakwe@gmail.com

${ }^{3}$ Department of Mathematics and Statistics, University of Port Harcourt, Nigeria

E-mail address: jerryezeora@yahoo.com 